\title{
Provision of Reproductive Healthcare to Women with Disabilities: \\ A Survey of Obstetrician-Gynecologists' Training, Practices, and Perceived Barriers
}

\author{
Laura H. Taouk, ${ }^{1,2}$ Michael F. Fialkow, ${ }^{3, *}$ and Jay A. Schulkin ${ }^{1,3}$
}

\begin{abstract}
Purpose: The purpose of this study was to document current awareness, attitudes, and training regarding the care of women with disabilities by obstetrician-gynecologists (ob-gyns) and explore barriers that may explain observed discrepancies in care.

Methods: One thousand ob-gyns, including 500 members of the Collaborative Ambulatory Research Network (CARN), were surveyed on practice accessibility, training, awareness, barriers, beliefs, comfort, challenges, practices, contraceptive counseling, and preconception/pregnancy counseling.

Results: CARN, 49.0\%, and non-CARN, 19.4\%, members completed the survey for an overall response rate of $33.9 \%$. Most respondents indicated feeling "somewhat" (57.5\%) or "very" (21.9\%) aware of the special healthcare needs of women with disabilities. Only $17.2 \%$, however, received any information or training on the provision of healthcare to women with disabilities. Eighty-one percent agreed somewhat or strongly that women with disabilities are less likely to receive comprehensive reproductive healthcare. Respondents who provided contraceptive counseling (94.3\%) initiated it with women of reproductive age who did not have a disability more frequently than those who had a disability. Finally, only $19.3 \%$ felt "definitely" adequately equipped to manage the pregnancies of women with disabilities.

Conclusion: Women with disabilities require reproductive healthcare no less than women without disabilities; however, the evidence consistently identifies disparities. This study suggests that while ob-gyn providers are aware of these issues, they lack adequate training and resources to provide equal care.
\end{abstract}

Keywords: obstetrician-gynecologists; survey; health disparity

\section{Introduction}

A disability can be defined as "a physical or mental impairment that substantially limits one or more major life activities." ${ }^{\prime \prime}$ More than 53 million adults in the United States reported a disability in 2013; mobility (13.0\%) and cognitive (10.6\%) disabilities were most common. $^{2}$ Enacted in 1990 to promote equal access to a range of services, the Americans with Disabilities Act (ADA) prohibits discrimination against persons with disabilities in healthcare settings, such that accessible facilities, aids for effective communication, and modified procedures to address special needs are mandated. ${ }^{1}$

Evidence is accumulating that women with disabilities receive healthcare screening less often than is recommended. Women with physical and cognitive disabilities are screened for breast cancer and cervical cancer less frequently than women without disabilities. ${ }^{3-5}$ Even

\footnotetext{
${ }^{1}$ Research Department, The American College of Obstetricians and Gynecologists (ACOG), Washington, District of Columbia.

${ }^{2}$ Department of Psychology, American University, Washington, District of Columbia.

${ }^{3}$ Department of Obstetrics and Gynecology, University of Washington School of Medicine, Seattle, Washington.

*Address correspondence to: Michael F. Fialkow, MD, MPH, Department of Obstetrics and Gynecology, University of Washington School of Medicine, 1959 NE Pacific Street, Seattle, WA 98195, E-mail: fialkow@gmail.com

(c) Laura H. Taouk et al. 2018; Published by Mary Ann Liebert, Inc. This Open Access article is distributed under the terms of the Creative Commons License (http://creativecommons.org/licenses/by/4.0), which permits unrestricted use, distribution, and reproduction in any medium, provided the original work is properly cited.
} 
after controlling for demographic and health-related characteristics, women with disabilities are found to be less likely to be up-to-date with mammograms, Pap tests, pelvic examinations, and clinical breast examinations. ${ }^{6-11}$ Data further suggest the more severe the disability, the less likely women are to be screened for breast and cervical cancer. ${ }^{3,9,12,13}$ In addition to lower screening rates, women with disabilities also report lower receipt of family planning services, including contraceptive counseling, and concern regarding physician preparedness to handle their pregnancies. ${ }^{14-16}$

Healthcare providers likely face structural, informational, and attitudinal challenges to caring for women with disabilities. ${ }^{17,18}$ Differences in health service utilization and insurance coverage do not explain apparent discrepancies in screening between women with and without disabilities. ${ }^{4,10,19}$ Furthermore, women with disabilities are less likely to receive a doctor's recommendation for screening, suggesting that structural and/or clinical factors underlie observed differences. ${ }^{4,10}$ Barriers to the provision of care may include physically inaccessible facilities, lack of communication aids, inadequate appointment lengths, and lack of provider knowledge regarding disabilities and modified screening practices. ${ }^{3,20,21}$ In addition, women with disabilities could be perceived as less likely to be sexually active and, consequently, healthcare providers may make inaccurate assumptions about their reproductive healthcare needs. ${ }^{20,21}$

Obstetrician-gynecologists (ob-gyns) are critical to the provision of effective healthcare for women. While discrepancies in care are well documented, reports on ob-gyns' perspectives regarding the provision of care to women with disabilities are relatively limited (e.g., small sample size, narrow scope). ${ }^{22}$ To address this, we surveyed a nationally representative sample of ob-gyns, belonging to the American College of Obstetricians and Gynecologists (ACOG), regarding the care of women with physical disabilities and intellectual or developmental disabilities (hereafter abbreviated as I/DD). The aims of this study were to (1) document current awareness, attitudes, and training regarding the care of women with disabilities (2) as well as the perceived educational and clinical barriers that may explain observed discrepancies in care.

\section{Methods}

Measures

A six-page questionnaire was developed and revised based on pretesting with five clinically active ob-gyns.
The first section of the survey assessed practice accessibility, training, awareness, barriers, and beliefs about the provision of care to women with disabilities. Sections two and three focused on comfort, challenges, and practices associated with the care of women with physical disabilities (defined as "conditions that limit physical functioning, mobility, dexterity, and/or stamina; e.g., muscular dystrophy, amputation, or sensory impairments") and I/DD (defined as "conditions that can affect language, learning, reasoning, problem solving, adaptive behaviors, and/or independent living; e.g., Down syndrome, fragile $\mathrm{X}$ syndrome, and cerebral palsy"). Subsequent sections assessed issues related to contraceptive counseling and preconception/pregnancy counseling. Demographic questions were included.

\section{Procedures}

The University of Washington Institutional Review Board determined the study to be of exempt status, due to the use of minimal risk survey procedures. Data collection began in November 2016. A random sample of 1,000 ACOG Fellows, 500 of whom belonged to the Collaborative Ambulatory Research Network (CARN), were invited to participate. CARN comprised Fellows who volunteer to participate in survey studies, and samples are found to be demographically representative of the greater ACOG membership. ${ }^{23}$ Ob-gyns were e-mailed links to the online questionnaire, along with information for informed participation, through the survey platform Qualtrics. Reminders were sent to nonresponders who had not opted out. In February, paper copies of the survey and prepaid return envelopes were mailed to nonresponders. Finally, an abbreviated version of the survey, which retained demographics and most items from the first three sections, was mailed to nonresponders. Data collection ended in May 2017.

\section{Participants}

Of those invited to participate, $49.0 \%$ of CARN and $19.4 \%$ of non-CARN members completed the survey for an overall response rate of $33.9 \%(n=322)$. Responses were excluded if the physician was no longer in practice $(n=1)$ or left at least $50 \%$ of the survey blank $(n=13)$ for a final sample of 308 ob-gyns. Half completed the survey online $(53.9 \% ; 46.1 \%$ on paper), and most completed the full-length version $(87.0 \% ; 13.0 \% \mathrm{ab}-$ breviated version). Sample demographics are described in Table 1. Males had been in practice longer $(m=28.6 \pm 8.4)$ than females $(m=19.0 \pm 9.3 ; t=9.03$, $p<0.001)$. Few ob-gyns (3.6\%) had a disability, while 
Table 1. Sample Demographics $(N=308)$

\begin{tabular}{lclc}
\hline Years in practice postresidency & $22.8 \pm 10.1$ & Age & $54.3 \pm 10.2$ \\
Gender (\%) & & Practice setting (\%) & Ob-gyn partnership/group \\
Female & 59.7 & University faculty and practice & 28.2 \\
Male & 38.6 & Hospital or clinic & 18.5 \\
Ethnicity/race (\%) & & Multispecialty group & 16.6 \\
White & 78.2 & Solo private practice & 14.6 \\
Black or African American & 6.5 & HMO/staff model & 13.6 \\
Asian & 6.5 & Military/government & 3.2 \\
Multiracial & 2.9 & Practice location (\%) & 2.3 \\
Hispanic or Latino & 2.3 & Suburban & 36.0 \\
Primary medical practice (\%) & & Urban, noninner city & 23.4 \\
General ob-gyn & 64.9 & Urban, inner city & 19.2 \\
Gynecology only & 24.7 & Midsized town $(10,000-50,000)$ & 12.3 \\
Obstetrics only & 8.4 & Rural & 6.8 \\
& & & \\
\hline
\end{tabular}

HMO, health maintenance organization; ob-gyn, obstetrician-gynecologist.

27.9\% had a close friend or family member with a disability. CARN members were slightly more likely to practice in urban areas and midsized towns than nonCARN participants $\left(\chi^{2}=10.00, p=0.040\right)$, however, other demographic differences were not found. Data were analyzed in aggregated form.

\section{Statistical analyses}

Data analysis was conducted using a personal computerbased software package (IBM SPSS Statistics ${ }^{\circledR}$ 23.0; IBM Corp $\odot$, Armonk, NY). Categorical response options endorsed by $<10 \%$ of the sample were collapsed; incremental, unidirectional response options were evaluated as continuous variables. Relationships between categorical variables were evaluated using chi-square tests. Relationships between continuous variables were evaluated using Pearson correlations, while group differences in continuous variables were evaluated using independentsamples and paired $t$-tests and ANOVAs. Linear regression models were also used to explore continuous and dichotomous predictors of continuous outcomes. All tests were considered significant at $p<0.05$, and valid percentages are reported.

\section{Results}

\section{Awareness and training}

Most ob-gyns indicated feeling "somewhat" (57.5\%) or "very" (21.9\%) aware of the special healthcare needs of women with disabilities. Greater awareness was related to more years in practice $(F=6.15, p=0.002)$; those who felt "very aware" were also more likely to have a close friend or relative with a disability $\left(\chi^{2}=13.82\right.$, $p=0.001)$ or received information or training $\left(\chi^{2}=19.73\right.$, $p<0.001$ ). Only $17.2 \%$ of ob-gyns had received any information or training on the provision of healthcare to women with disabilities. Information or training on facilitating pelvic examinations for patients with mobility-limited physical disabilities (88.9\%) and modifying communication approach for those with visual, hearing, or cognitive disabilities (71.1\%) were most common; preventing autonomic reactions to an examination was least common (28.9\%). Receipt of training/ information was less likely for ob-gyns in private practice or a partnership/group $\left(\chi^{2}=13.60, p=0.018\right)$, but unrelated to years in practice. Only $4.8 \%$ reported additional training would be "not beneficial at all."

\section{Provision of care and barriers}

Most ob-gyns (81\%) agreed somewhat or strongly that "women with disabilities are less likely to receive comprehensive reproductive healthcare than women without disabilities." Only $2.3 \%$ rated the provision of comprehensive healthcare for women with disabilities as "not challenging at all." Barriers are listed in Table 2. Average barrier scores $(\alpha=0.794 ; m=1.71 \pm 0.42)$ were lower for ob-gyns whose practice was handicap accessible (e.g., ramps, elevators, wide doors; $94.4 \%$; $t=-2.52$, $p=0.012$ ), had at least one examination room with adapted medical equipment to accommodate persons with disabilities (e.g., adjustable-height examination tables; $86.2 \% ; \mathrm{t}=-2.25, p=0.025$ ), and had resources to communicate with patients with vision or hearing impairments (e.g., paperwork in Braille, ASL speaker; 42.2\%; $F=6.10, p=0.003)$. Ob-gyns in solo private practice or a partnership/group were more likely to endorse the starred items as major barriers (Table 2: $\chi^{2}=21.37$, $p=0.019 ; \chi^{2}=20.17, p=0.028 ; \chi^{2}=27.76, p=0.002$ ) and less likely to have an accessible examination room $\left(\chi^{2}=44.70, p<0.001\right)$ or communication resources $\left(\chi^{2}=82.62, p<0.001\right)$. 
Table 2. Barriers to the Provision of Care

\begin{tabular}{|c|c|c|c|}
\hline & $\begin{array}{c}\text { Not a } \\
\text { barrier (\%) }\end{array}$ & $\begin{array}{c}\text { Minor } \\
\text { barrier (\%) }\end{array}$ & $\begin{array}{c}\text { Major } \\
\text { barrier (\%) }\end{array}$ \\
\hline Inaccessible office location and equipment & 76.3 & 18.8 & 4.2 \\
\hline Limited insurance reimbursement for extra time and care provided* & 39.9 & 32.5 & 27.3 \\
\hline Difficulty with positioning during examinations & 27.6 & 54.5 & 17.5 \\
\hline Fear of autonomic dysreflexia or other autonomic reactions to the examination & 51.1 & 41.4 & 6.3 \\
\hline Fear of causing patients discomfort, pain, or embarrassment & 56.5 & 36.0 & 7.1 \\
\hline Inadequate knowledge about specific disabilities and special needs* & 31.5 & 54.2 & 14.0 \\
\hline Uncertainty regarding appropriate sexual and reproductive recommendations & 56.3 & 34.7 & 9.0 \\
\hline Difficulty communicating with patients who have visual, hearing, or cognitive disabilities* & 28.2 & 48.1 & 23.7 \\
\hline $\begin{array}{l}\text { Uncertainty about decision-making capacities or consent to medical procedures } \\
\text { with patients who have intellectual or developmental disabilities }\end{array}$ & 28.6 & 50.0 & 21.4 \\
\hline
\end{tabular}

Responses to the question stem: "in your practice, what are the barriers to the provision of healthcare for women with disabilities?" ( $N=308)$. Each item was rated as "not a barrier," a "minor barrier," or a "major barrier". Italicized items were not included on the abbreviated survey ( $N=268)$. Starred items were more likely to be endorsed as major barriers by ob-gyns in private practice or a partnership/group.

\section{Comfort and confidence}

In a typical month, ob-gyns estimated they saw an average of $4.3( \pm 20.9)$ patients with a physical disability and $4.2( \pm 4.1)$ with an I/DD. For women with physical disabilities, most ob-gyns were "very comfortable" asking about gynecological, sexual, and reproductive history (68.5\%); performing a pelvic examination (65.9\%); performing a breast examination (80.0\%); and managing sexual/reproductive care $(58.0 \%)$. For women with an $\mathrm{I} / \mathrm{DD}$, fewer ob-gyns were "very comfortable" with these parts of well-women care (respectively: 50.0\%, $46.7 \%, 58.4 \%, 44.7 \%)$. Greater average comfort ratings $(\alpha=0.913, B=0.26 ; \alpha=0.936 ; B=0.41)$, receipt of information or training $(B=0.23 ; B=0.29)$, and fewer barriers to the provision of care $(B=-0.77 ; B=-0.67)$ predicted higher physician confidence in their ability to provide appropriate care for women with physical disabilities $\left(F=29.88, p<0.001 ; R^{2}=0.288\right)$ and $\mathrm{I} / \mathrm{DD}$ $\left(F=61.05, \quad p<0.001 ; R^{2}=0.380\right)$. Confidence ratings are shown in Figure 1. Years in practice and number of patients seen were not associated with comfort nor confidence providing care.

\section{Practices}

For mobility-limited patients, most ob-gyns never (43.2\%) or only sometimes (50.4\%) performed examinations with women remaining in their mobility device. However, a portion of respondents indicated they "never" examined high-pressure areas of skin for decubitus ulcers or pressure sores (29.9\%) nor recommend earlier screening for osteoporosis $(27.9 \%)$ or cardiac health $(41.2 \%)$. For patients with limited bodily sensation, $60.1 \%$ of ob-gyns reported they "never" provided information about alternative (nonsensation based) symptoms of breast and cervical cancer. If clinically indicated pelvic examinations could not be accom- modated, most ob-gyns preferred to recommend an examination under sedation or anesthesia (34.7\%) or an ultrasound (32.2\%). For patients with an $\mathrm{I} / \mathrm{DD}$, some ob-gyns reported they "most of the time" or "always" primarily communicated with the patient's guardian $(49.0 \%)$ and deferred to the guardian to make health decisions in situations of shared decision-making status (38.0\%). Half (51.1\%) reported mostly or always using supported decision-making strategies to assist patients in making decisions.

\section{Contraceptive counseling}

Ob-gyns who provided contraceptive counseling $(94.3 \%)$ initiated it with women of reproductive age who did not have a disability more frequently than those who had a physical disability $(t=5.68, p<0.001)$ or an I/DD ( $t=7.03, p<0.001$; Fig. 2$)$. However, most "strongly" disagreed that women with a physical disability $(59.8 \%)$ or an I/DD (64.3\%) were less likely to require contraceptive counseling. Barriers are listed in Table 3. Lower barrier scores $(m=2.0 \pm 0.5 ; \alpha=0.878)$ related to more frequent initiation of contraceptive counseling for women with physical disabilities $(r=-0.14$, $p=0.025)$ and I/DD $(r=-0.17, p=0.009)$ and feeling more adequately equipped to manage contraceptive care of women with disabilities (30.2\% "definitely yes," $56.3 \%$ "probably yes," $13.4 \%$ "probably or definitely not"; $F=20.80, p<0.001)$. Ob-gyns who were aware of any guidelines on contraceptive counseling for women with disabilities $(24.9 \%)$ were more likely to feel adequately equipped $\left(\chi^{2}=10.53, p=0.005\right)$ and rank intrauterine devices as a top three contraception recommendation for women with physical disabilities $\left(\chi^{2}=4.28, p=0.039\right)$ and I/DD $\left(\chi^{2}=5.76, p=0.016\right.$; Fig. 3). Sterilization was more likely to be a top three recommendation for women with I/DD $(t=-2.99$, 


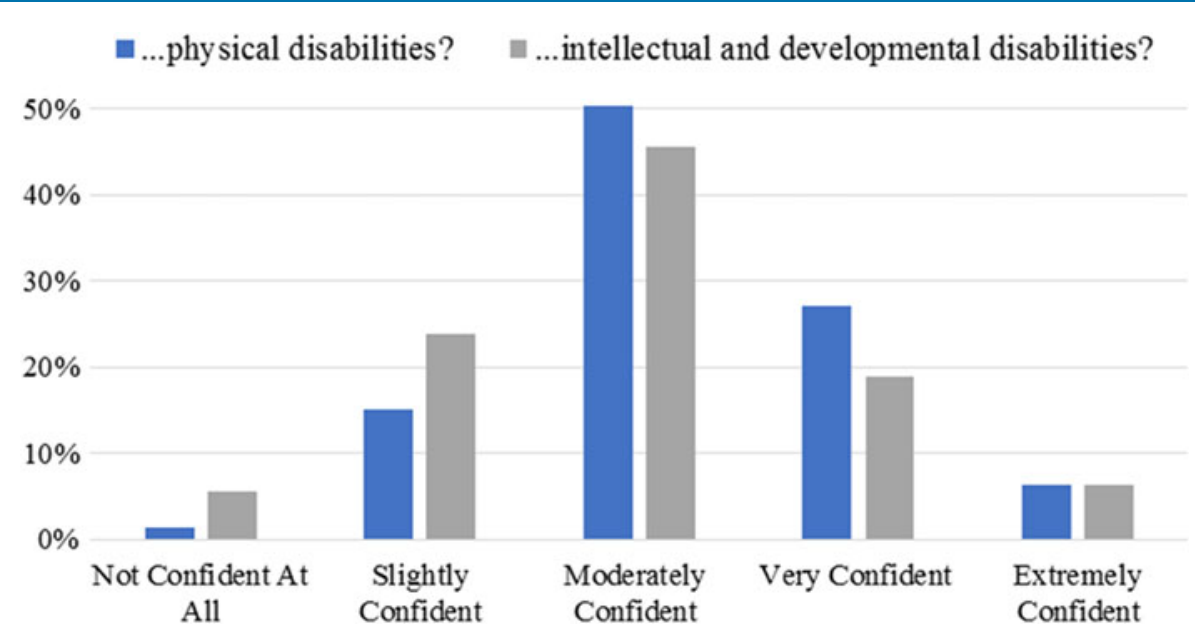

FIG. 1. Confidence in ability to provide care to women with disabilities. Responses to, "how confident are you in your ability to provide appropriate healthcare for women with... [(1) physical disabilities; (2) intellectual and developmental disabilities]," were rated on a scale from "not confident at all" to "extremely confident" $(N=304)$.

$p=0.003)$, but not physical disabilities $(t=-1.26$, $p=0.209)$, compared with women without disabilities.

\section{Preconception and pregnancy counseling}

Of the $90.0 \%$ of ob-gyns who counseled patients considering pregnancy, 29.5\% "most of the time" or "always" referred patients with disabilities to a specialist. Those who provided preconception counseling most frequently emphasized potential difficulties with the pregnancy (93.5\%), difficulties with labor and delivery $(88.7 \%)$, and genetic effects, or consultation with a genetic counselor (84.8\%). Potential resources to support the transition into pregnancy and

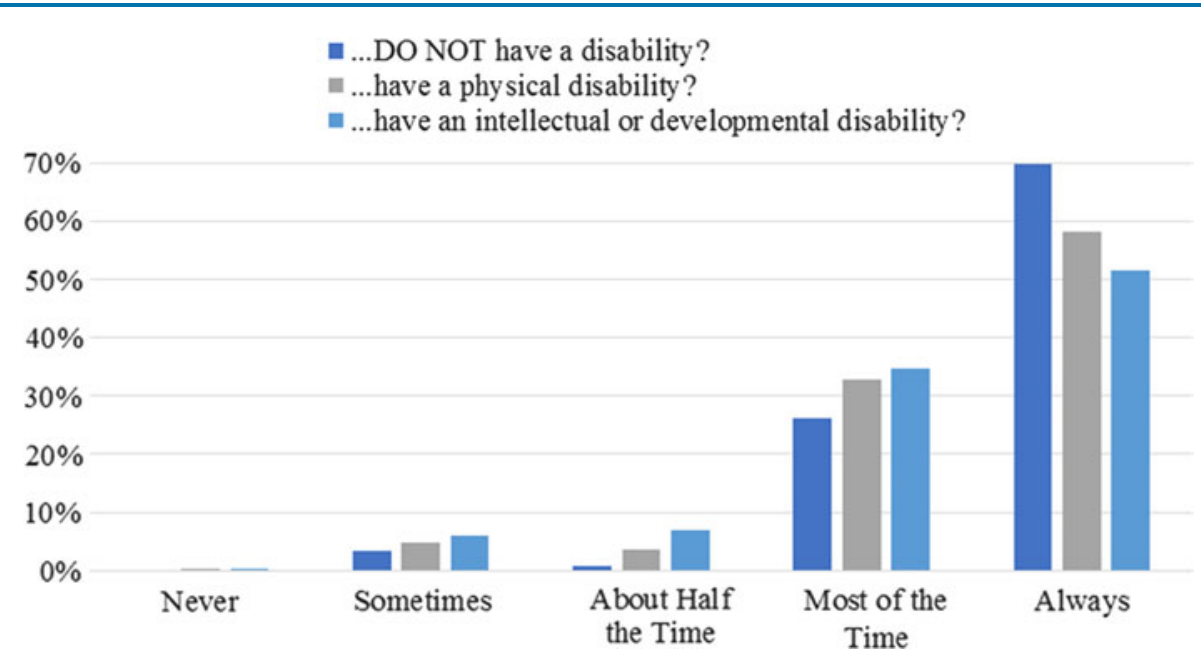

FIG. 2. Frequency of initiating contraceptive counseling. Responses to, "in your current practice, how often do you initiate contraceptive counseling with women of reproductive age who...[(1) do not have a disability; (2) have a physical disability; (3) have an intellectual or developmental disability]," were rated on a scale from "never" to "always." Items were answered by full-length survey respondents who provided contraceptive counseling $(N=244)$. 


\begin{tabular}{lccr}
\hline & Not a barrier (\%) & Minor barrier (\%) & Major barrier (\%) \\
\hline Determining whether patients require contraceptive counseling & 56.3 & 39.2 & 4.1 \\
Determining ability to independently and properly utilize contraception & 24.1 & 49.0 & 26.9 \\
Determining decision-making capacities for contraception and sex & 20.8 & 49.0 & 29.0 \\
Determining consent to irreversible means of contraception & 17.1 & 46.5 & 36.3 \\
Determining patient understanding of contraception risks and benefits & 13.9 & 58.4 & 27.8 \\
Determining patient understanding of STD or pregnancy prevention & 13.5 & 58.8 & 27.8
\end{tabular}

Responses to the question stem: "in your practice, what are the barriers to providing contraceptive counseling for women with disabilities?" Each item was rated as "not a barrier," a "minor barrier," or a "major barrier." Items were answered by full-length survey respondents who provided contraceptive counseling $(N=245)$.

STD, sexually transmitted disease.

parenthood were least frequently emphasized (54.5\%). Only $19.3 \%$ of physicians felt "definitely" adequately equipped to manage the pregnancies of women with disabilities (52.4\% "probably yes"; $20.2 \%$ "probably not"; $8.2 \%$ "definitely not"). Nearly all ob-gyns (92.2\%) endorsed a need for more informational resources to help physicians guide women with disabilities through pregnancy, its management, and the transition into parenthood.

\section{Discussion}

Women with disabilities require reproductive healthcare no less than women without disabilities; however, access to skilled and culturally competent care, where special needs are recognized and addressed, remains an obstacle. Although evidence points to persistent disparities in women's reproductive care, provider perspectives remain sparse in the literature. This study evaluated practicing ob-gyns' comfort, opinions, and

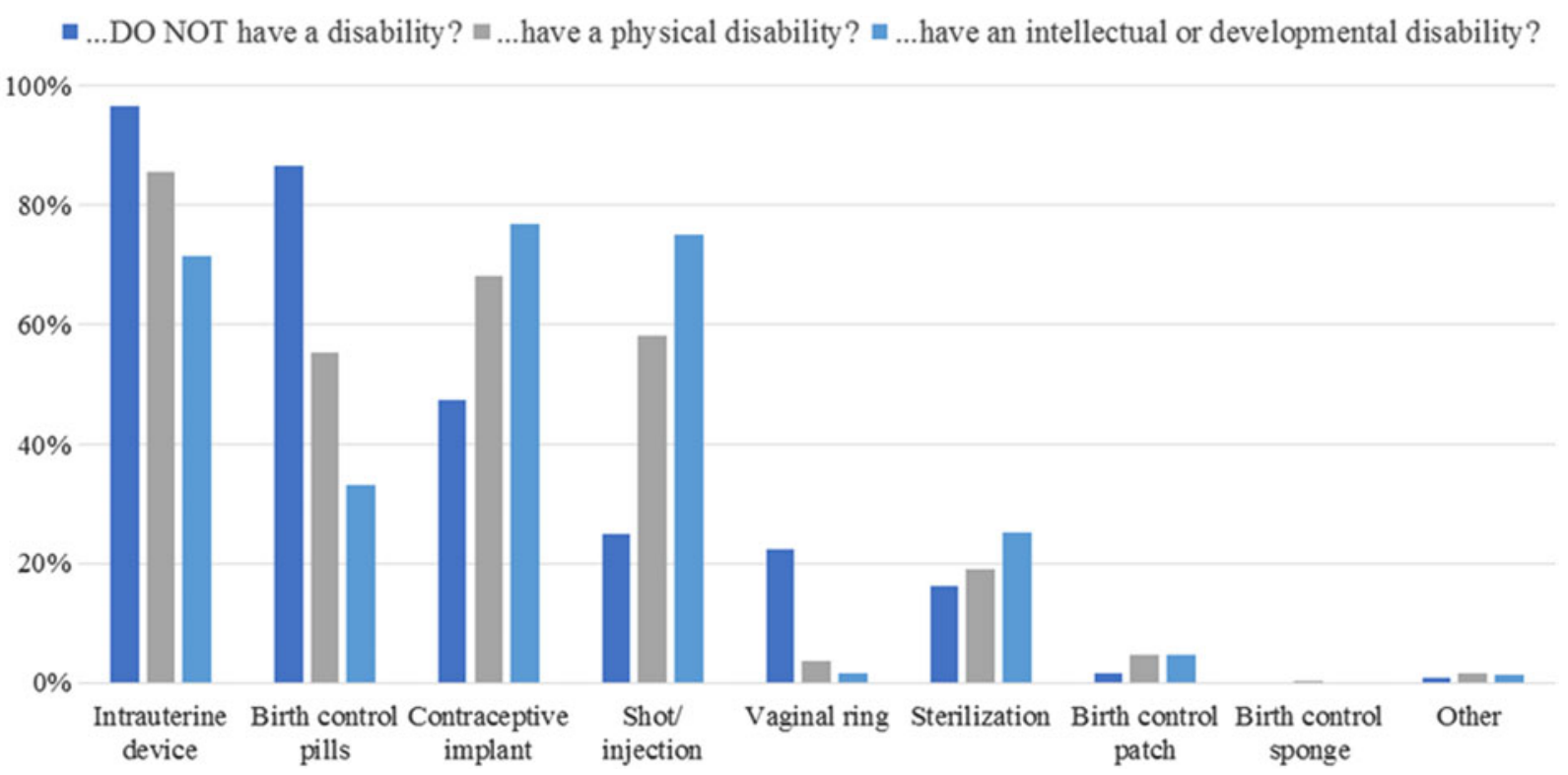

FIG. 3. Top three contraception recommendations. Responses to, "please rank the top three types of contraception that you most often recommend for patients who...[(1) do not have a disability; (2) have a physical disability; (3) have an intellectual or developmental disability]. Items were answered by full-length survey respondents who provided contraceptive counseling $(N=241)$. Since many respondents did not provide numbered rankings (e.g., writing \#2 next to their second choice), responses were recoded to reflect options that were selected as top three recommendations. 
practices regarding the provision of well-woman, gynecological, and obstetric healthcare to women with disabilities. Findings highlight barriers perceived by ob-gyns, as well as educational and structural needs, which may inform future efforts toward reproductive health equity.

Supporting evidence of disparities, $81 \%$ of ob-gyns in our sample believed women with disabilities are less likely to receive comprehensive reproductive healthcare. ${ }^{3,4}$ While most ob-gyns reportedly had handicap accessible practices, less than half had communication resources, and $71.8 \%$ endorsed "difficulty communicating with patients who have visual, hearing, or cognitive disabilities." Although required by ADA, there is evidence to suggest many subspecialty practices cannot accommodate patients with mobility impairments and auxiliary aids/services are still lacking. ${ }^{20,24,25}$ Other common barriers (Table 2) implicate inadequate knowledge as a prominent challenge faced by physicians, consistent with prior findings. ${ }^{26}$

Nearly all ob-gyns indicated additional training would be at least slightly beneficial. Only $17.2 \%$ had received any information or training on the provision of healthcare to women with disabilities and far fewer on specific issues such as preventing autonomic dysreflexia, a potentially life-threatening condition that can occur during gynecological examinations of women with spinal cord injuries. ${ }^{18}$ Higher training rates have been found in a general physician sample $(22.8 \%$, $34.1 \%){ }^{27}$ The Accreditation Council for Graduate Medical Education (ACGME) indicates ob-gyn residents are "expected to demonstrate sensitivity and responsiveness to a diverse patient population, including ... disabilities," but didactic requirements are absent. ${ }^{28}$

Despite barriers and training deficits, most ob-gyns felt aware of the special healthcare needs of women with disabilities, and few felt uncomfortable with components of an examination for women with physical disabilities $(\leq 6.5 \%)$ or I/DD $(\leq 12.8 \%)$. Greater gynecologist discomfort was endorsed in a decade-old study (14-42\%). ${ }^{26}$ While ob-gyns likely attempt to accommodate patients as best they can, some reported practices were concerning. For instance, when treating patients with mobility limitations, around one in three ob-gyns reportedly never examined high-pressure areas of skin for decubitus ulcers or pressure sores, which are common sources of increased pain and morbidity, nor recommended early bone density or cardiovascular disease screening, despite increased risk. ${ }^{3,29,30}$ In addition, only half of ob-gyns endorsed using supported decision-making strategies most or all the time for patients with $\mathrm{I} / \mathrm{DD}$, despite their utility in protecting patients' rights and assisting them in making health decisions. ${ }^{31}$

Regarding contraceptive counseling, it was reportedly initiated more frequently with nondisabled patients, even though few ob-gyns $(<9 \%)$ indicated women with disabilities were less likely to require it. This discrepancy between reported practice and opinion could suggest ob-gyns harbor implicit stereotypes of women with disabilities as asexual, which leads to biased provision of care. Alternatively, or additionally, this discrepancy could be explained by nonattitudinal barriers. Except for "determining whether patients require contraceptive counseling," each barrier was endorsed by more than $75 \%$ of the sample (Table 3 ). Counseling requires special considerations, such as ability to provide informed consent and independently utilize contraception, yet only one in four ob-gyns was aware of any guidelines on contraceptive counseling for women with disabilities. ${ }^{18}$ Although experts recommend against irreversible contraception, a substantial number of ob-gyns selected sterilization as one of the top three types of contraception they most often recommended for patients with physical disabilities (19.1\%) or I/DD (25.3\%; Fig. 3). ${ }^{18,32}$ Encouragingly, rank-ordered responses indicated sterilization was rarely ranked as the first-choice recommendation (physical disabilities: 0\%, I/DD: 2.7\%).

During preconception counseling, ob-gyns emphasized many potential patient concerns, but only half emphasized resources to support the transition into pregnancy and parenthood. Nearly all ob-gyns acknowledged a need for more informational resources to help physicians guide women with disabilities through pregnancy, its management, and the transition into parenthood, as previously proposed. ${ }^{14}$ Only $19.3 \%$ of ob-gyns felt "definitely" adequately equipped to manage the pregnancies of women with disabilities. This is consistent with patient reports that clinicians are ill equipped to manage their pregnancies effectively. ${ }^{14}$ Such findings are alarming, given evidence that women with disabilities, particularly I/DD, are at a greater risk for pregnancy and birth complications, as well as adverse pregnancy outcomes. ${ }^{33-35}$

The present study examined the provision of reproductive healthcare to women with disabilities in a representative sample of practicing ob-gyns, contributing much needed data on provider needs and perspectives. However, this study had several limitations. To begin 
with, the sample size was lower than hoped for, and therefore, results may not ideally represent practicing ob-gyns. Second, the survey was designed to obtain a general understanding of the issues for ob-gyns, since little is currently known. Complementary questions regarding the care of nondisabled women were not always included, constricting exploration of whether responses reflect disability-specific opinions, attitudes, comfort, and practices. Some terms (e.g., "accessible") were not defined, which could have affected responses. Furthermore, it is possible that social desirability bias influenced responses to questions regarding opinions and attitudes. Self-reported practices may also differ from actual care delivered, and interpretation of reported practices is limited, as individualized, case-by-case considerations cannot be known. Considering physical disabilities and I/DD encompass a wide range of presentations, physicians potentially responded to questions with varying levels of disability severity in mind. Finally, questionnaire-based data preclude the possibility of drawing causal conclusions.

\section{Conclusion}

This study is hopefully a first step toward improving quality of care for women with disabilities. Findings indicate training deficits, inadequate knowledge, and unawareness of guidelines could be significant barriers preventing ob-gyns from providing comprehensive reproductive healthcare to women with disabilities. Results also suggest ob-gyns' confidence in their ability to provide appropriate care could be improved by targeting training/information, practice barriers, and comfort with components of the examination that may require modified approach. Training physicians to meet the special (e.g., cervical and breast cancer screening) needs of women with disabilities is a federally recognized issue. ${ }^{21}$ Education and resources are needed to support physicians and patients with disabilities and promote comfort, respect, and safety. ${ }^{18,32}$

Going forward, it is imperative that educational initiatives, as well as clinical guidelines, are developed, empirically evaluated, and disseminated to reproductive healthcare practitioners. Materials, such as "The Toolbox" and ACOG's recorded slide program, are available to facilitate the implementation of care; however, their utilization, reach, and relevance to practitioners are presently unknown. ${ }^{36,37}$ Data are critically needed to "inform policy and program development regarding critical issues of health disparities and health equity," and systemic change in healthcare de- livery may ultimately be necessary. ${ }^{38,39}$ Our hope is that the data presented here will contribute to greater awareness of the barriers encountered by ob-gyns in their practice, informing future efforts toward the goal of equity in reproductive healthcare.

\section{Acknowledgments}

The authors thank Neko Castleberry and Carrie Snead for their assistance with survey revisions, data collection, and data entry, as well as their support of the study.

\section{Author Disclosure Statement}

No competing financial interests exist.

\section{References}

1. Americans with Disabilities Act of 1990, Pub. L. No. 101-336, 104 Stat. 328 United States. Washington, D.C. U.S. Department of Justice, Civil Rights Division, Disability Rights Section, 1990.

2. Courtney-Long EA, Carroll DD, Zhang QC, et al. Prevalence of disability and disability type among adults-United States, 2013. Morb Mortal Wkly Rep. 2015;64:777-783.

3. Smeltzer SC. Preventive health screening for breast and cervical cancer and osteoporosis in women with physical disabilities. Fam Community Health. 2006:29:35S-43S.

4. Parish SL, Saville AW. Women with cognitive limitations living in the community: evidence of disability-based disparities in health care. Ment Retard. 2006;44:249-259.

5. Wei W, Findley PA, Sambamoorthi U. Disability and receipt of clinical preventive services among women. Womens Health Issues. 2006;16: 286-296.

6. Reichard A, Stolzle H, Fox MH. Health disparities among adults with physical disabilities or cognitive limitations compared to individuals with no disabilities in the United States. Disability Health J. 2011;4:59-67.

7. Horner-Johnson W, Dobbertin K, lezzoni LI. Disparities in receipt of breast and cervical cancer screening for rural women age 18 to 64 with disabilities. Womens Health Issues. 2015;25:246-253.

8. Nosek MA, Howland C, Rintala DH, et al. National study of women with physical disabilities. Sex Disabil. 2001;19:5-40.

9. Diab ME, Johnston MV. Relationships between level of disability and receipt of preventive health services. Arch Phys Med Rehabil. 2004;85: 749-757.

10. Ramirez A, Farmer GC, Grant D, et al. Disability and preventive cance screening: results from the 2001 California Health Interview Survey. Am J Public Health 2005;95:2057-2064.

11. Brown HK, Plourde N, Ouellette-Kuntz $\mathrm{H}$, et al. Brief report: cervical cancer screening in women with intellectual and developmental disabilities who have had a pregnancy. J Intellect Disabil Res. 2016;60:22-27.

12. Andresen EM, Peterson-Besse JJ, Krahn GL, et al. Pap, mammography, and clinical breast examination screening among women with disabilities: a systematic review. Womens Health Issues. 2013;23:e205-e214.

13. lezzoni LI, McCarthy EP, Davis RB, et al. Use of screening and preventive services among women with disabilities. Am J Med Qual. 2001;16: 135-144.

14. Mitra M, Clements KM, Zhang J, et al. Maternal characteristics, pregnancy complications, and adverse birth outcomes among women with disabilities. Med Care. 2015:53:1027-1032.

15. Mosher W, Bloom T, Hughes R, et al. Disparities in receipt of family planning services by disability status: new estimates from the National Survey of Family Growth. Disabil Health J. 2017;10:394-399.

16. Smeltzer SC, Mitra M, lezzoni LI, et al. Perinatal experiences of women with physical disabilities and their recommendations for clinicians. J Obstet Gynecol Neonatal Nurs. 2016;45:781-789.

17. Schopp LH, Sanford TC, Hagglund KJ, et al. Removing service barriers for women with physical disabilities: promoting accessibility in the gynecologic care setting. J Midwifery Womens Health. 2002;47:74-79. 
18. Kaplan C. Special issues in contraception: caring for women with disabilities. J Midwifery Womens Health. 2006;51:450-456.

19. Wisdom JP, McGee MG, Horner-Johnson W, et al. Health disparities between women with and without disabilities: a review of the research. Soc Work Public Health. 2010;25:368-386.

20. Thierry JM. Observations from the CDC: increasing breast and cervical cancer screening among women with disabilities. J Womens Health Gend Based Med. 2000;9:9-12.

21. Peacock G, lezzoni LI, Harkin TR. Health care for Americans with disabilities-25 years after the ADA. N Engl J Med. 2015; 373:892-893.

22. Mitra M, Smith LD, Smeltzer SC, et al. Barriers to providing maternity care to women with physical disabilities: perspectives from health care practitioners. Disabil Health J. 2017;10:445-450.

23. England LJ, Anderson BL, Mahoney J, et al. Screening practices and attitudes of obstetricians-gynecologists toward new and emerging tobacco products. Am J Obstet Gynecol. 2014;211:695.e1-695.e7.

24. Grabois EW, Nosek MA, Rossi CD. Accessibility of primary care physicians' offices for people with disabilities: an analysis of compliance with the Americans with disabilities act. Arch Fam Med. 1999;8:44-51.

25. Lagu T, Hannon NS, Rothberg MB, et al. Access to subspecialty care for patients with mobility impairment: a survey. Ann Intern Med. 2013;158: 441-446.

26. Shah P, Norlin C, Logsdon V, et al. Gynecological care for adolescents with disability: physician comfort, perceived barriers, and potential solutions. J Pediatr Adolesc Gynecol. 2005;18:101-104.

27. McNeal MA, Carrothers LA, Premo B. Providing Primary Health Care for People with Physical Disabilities: A Survey of California Physicians. Pomona, CA: Center for Disability Issues and the Health Professions, Western University of Health Sciences, 2002.

28. Accreditation Council for Graduate Medical Education, 2013. ACGME program requirements for graduate medical education in obstetrics and gynecology. Accreditation Council for Graduate Medical Education website. 2013. Available at https://www.acgme.org/Portals/0/PFAssets/ ProgramRequirements/220_obstetrics_and_gynecology_2017-07-01.pdf Accessed February 18, 2018.

29. Melzer D, Gardener E, Guralnik JM. Mobility disability in the middle-aged: cross-sectional associations in the English Longitudinal Study of Ageing. Age Ageing. 2005;34:594-602.

30. Anders J, Heinemann A, Leffmann C, et al. Decubitus ulcers: pathophysiology and primary prevention. Dtsch Ärztebl Int. 2010;107:371.

31. Kohn NA, Blumenthal JA, Campbell AT. Supported decision-making: a viable alternative to guardianship. Penn State Law Rev. 2013;117: 1111-1157.
32. Abells D, Kirkham YA, Ornstein MP. Review of gynecologic and reproductive care for women with developmental disabilities. Curr Opin Obstet Gynecol. 2016;28:350-358.

33. Mitra M, Clements KM, Zhang J, et al. Disparities in adverse preconception risk factors between women with and without disabilities. Matern Child Health J. 2016;20:507-515.

34. Parish SL, Mitra M, Son E, et al. Pregnancy outcomes among US women with intellectual and developmental disabilities. Am J Intellect Dev Disabil. 2015;120:433-443.

35. Signore C, Spong CY, Krotoski D, et al. Pregnancy in women with physical disabilities. Obstet Gynecol. 2011;117:935-947.

36. Sinclair LB, Taft KE, Sloan ML, et al. Tools for improving clinical preventive services receipt among women with disabilities of childbearing ages and beyond. Matern Child Health J. 2015;19:1189-1201.

37. American College of Obstetricians and Gynecologists (ACOG). Reproductive health care for women with disabilities. Recorded slide program. Available at http://cfweb.acog.org/womenwithdisabilities/index.html Accessed December 12, 2017.

38. Office of Disease Prevention and Health Promotion (ODPHP). Disability and health. In healthy people 2020. 2016. Available at https:// www.healthypeople.gov/2020/topics-objectives/topic/disabilityand-health Accessed January 27, 2018.

39. Yee S, Breslin ML. Achieving accessible health care for people with disabilities: why the ADA is only part of the solution. Disabil Health J. 2010;3: 253-261.

Cite this article as: Taouk LH, Fialkow MF, Schulkin JA (2018) Provision of reproductive healthcare to women with disabilities: a survey of obstetrician-gynecologists' training, practices, and perceived barriers, Health Equity 2:1, 207-215, DOI: 10.1089/heq.2018.0014.

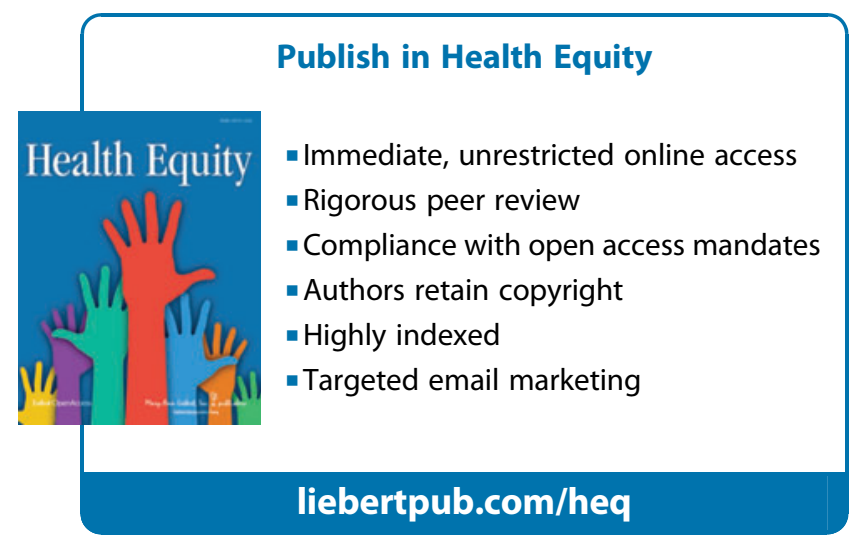

